\title{
ANALYSIS OF A PDE MODEL FOR SANDPILE GROWTH
}

\author{
P. Cannarsa ${ }^{1}$ \\ ${ }^{1}$ Department of Mathematics, University of Rome "Tor Vergata", Rome, Italy, \\ cannarsa@axp.mat.uniroma2,it
}

\begin{abstract}
In the dynamical theory of granular matter, the so-called table problem consists in studying the evolution of a heap of matter poured continuously onto a bounded domain $\Omega \subset \mathbf{R}^{2}$. The mathematical description of the table problem, at an equilibrium configuration, can be reduced to a boundary value problem for a system of partial differential equations. The analysis of such a system, also connected with other mathematical models such as the Monge-Kantorovich problem, is the object of this paper. Our main result is an integral representation formula for the solution, in terms of the boundary curvature and of the normal distance to the cut locus of $\Omega$.
\end{abstract}

keywords: granular matter, eikonal equation, singularities, viscosity solutions, optimal mass tranfer

\section{A PDE model for sandpile growth}

In recent years, the attention of many authors has been focussed on the system of partial differential equations

$$
-\operatorname{div}(v D u)=f \quad \text { in } \Omega, \quad|D u|-1=0 \quad \text { in }\{v>0\}
$$

in a given domain $\Omega \subset \mathbf{R}^{n}$.

For $n=2$, a typical context of application for (1) is granular matter theory. The so-called 'table problem', for instance, consists of describing the evolution of a sandpile created by pouring dry matter onto a table. Different approaches to this problem have been proposed in the literature, such as: the variational model developed by Prigozhin [20]; the ODE/PDE Model introduced in [2] and [14] by Evans and co-authors; the BCRE Model initiated by Boutroux and de Gennes[3] and elaborated by Hadeler and Kuttler[17]. In our analysis, we shall be concerned with the BCRE model, where the table is represented by a bounded domain $\Omega \subset \mathbf{R}^{2}$, and the matter source by a function $f(t, x) \geq 0$. The physical description of the growing heap is based on the introduction of the so-called standing and rolling layers. The former collects the amount of

Please use the following format when citing this chapter:

Author(s) [insert Last name, First-name initial(s)], 2006, in IFIP International Federation for Information Processing, Volume 199, System Modeling and Optimization, eds. Ceragioli F, Dontchev A., Furuta H., Marti K., Pandolfi L., (Boston: Springer), pp. [insert page numbers]. 
matter that remains at rest, the latter represents matter moving down along the surface of the standing layer-eventually falling down when the base of the heap touches the boundary of the table.

As pointed out in [17], system (1) is related to equilibrium configurations that may occur in presence of a constant source. To explain this connection, let us denote by $u(x)$ and $v(x)$, respectively, the heights of the standing and rolling layers at a point $x \in \Omega$, for an equilibrium configuration. For physical reasons, the slope of the standing layer cannot exceed a given constant (the angle of repose)-typical of the matter under consideration-that we normalize to 1 . Consequently, the standing layer must vanish on the boundary of the table. So, $|D u| \leq 1$ in $\Omega$ and $u=0$ on $\partial \Omega$. Also, in the region where $v$ is positive, the standing layer has to be 'maximal', for otherwise more matter would roll down there to rest. On the other hand, the rolling layer results from transporting matter, poured by the source, along the surface of the standing layer at a speed that is assumed proportional to the slope $D u$, with constant equal to 1 . The above considerations lead to the boundary value problem

$$
\begin{cases}-\operatorname{div}(v D u)=f & \text { in } \Omega \\ |D u|-1=0 & \text { in }\{v>0\} \\ |D u| \leq 1 \quad u, v \geq 0 & \text { in } \Omega \\ u=0 & \text { on } \partial \Omega\end{cases}
$$

Notice that (2) is the same equilibrium system one would obtain from Prigozhin's variational model.

\subsection{Connection with optimal mass transfer}

It is worth noting that system (1) arises in Monge-Kantorovich theory, as explained in the monograph [15], and futher analyzed in [1] and [16]. In the present context, we will just observe that the connection of the above system with optimal mass transfer can be obtained by looking at the so-called 'dual problem', which consists in maximizing

$$
\int_{\Omega} u(x) f(x) d x
$$

among all Lipschitz continuous functions $u: \Omega \rightarrow \mathbf{R}$, with $\operatorname{Lip}(u) \leq 1$, vanishing on $\partial \Omega$. Indeed, as proved in [4], the boundary value problem (2) turns out to be the system of necessary conditions satisfied by any maximizer $u$ of (3), taking $v$ equal to the associated Lagrange multiplier. Such a framework is also related to the optimization problem studied in [12].

\subsection{Solution of the equilibrium system}

The main purpose of the present work is to provide a full analysis of problem (2), including existence, uniqueness, and regularity of the solution. For 
existence and uniqueness, we shall follow the approach of [5] for the case of $n=2$, and of [6] for the general case $n \geq 2$. As for regularity, we shall rely on the results of [7]. It is well-known that the eikonal equation

$$
|D u|=1
$$

does not possess global smooth solutions in general, neither does the conservation law

$$
-\operatorname{div}(v D u)=f
$$

Therefore, we ought to explain what we mean by a solution of (2).

We say that a pair $(u, v)$ of continuous functions in $\Omega$ is a solution of problem (2) if

- $u=0$ on $\partial \Omega,\|D u\|_{\infty, \Omega} \leq 1$, and $u$ is a viscosity solution of

$$
|D u|=1 \quad \text { in } \quad\{x \in \Omega: v(x)>0\}
$$

- $v \geq 0$ in $\Omega$ and, for every test function $\phi \in \mathcal{C}_{c}^{\infty}(\Omega)$,

$$
\int_{\Omega} v(x)\langle D u(x), D \phi(x)\rangle d x=\int_{\Omega} f(x) \phi(x) d x .
$$

For the reader's sake, we now recall the definition of viscosity solution. The superdifferential of a function $u: \Omega \rightarrow \mathbf{R}$ at a point $x \in A$ is the set

$$
D^{+} u(x)=\left\{p \in \mathbf{R}^{\mathbf{n}} \mid \limsup _{h \rightarrow 0} \frac{u(x+h)-u(x)-\langle p, h\rangle}{|h|} \leq 0\right\},
$$

while the subdifferential $D^{-} u$ is given by the formula $D^{-} u(x)=-D^{+}(-u)(x)$. We say that $u$ is a viscosity solution of the eikonal equation $|D u|=1$ in $\Omega$ if, for any $x \in \Omega$, we have

$$
p \in D^{-} u(x) \Rightarrow|p| \geq 1, \quad p \in D^{+} u(x) \Rightarrow|p| \leq 1 .
$$

Before describing our main results, we need to introduce some useful geometric properties of bounded domains with smooth boundary. This is the purpose of the next section.

\section{Distance function}

Let $\Omega$ be a bounded domain with $\mathcal{C}^{2}$ boundary $\partial \Omega$. In what follows we denote by $d: \bar{\Omega} \rightarrow \mathbf{R}$ the distance function from the boundary of $\Omega$, that is,

$$
d(x)=\min _{y \in \partial \Omega}|y-x|
$$


and by $\Sigma$ the singular set of $d$, that is, the set of points $x \in \Omega$ at which $d$ is not differentiable. Since $d$ is Lipschitz continuous, $\Sigma$ has Lebesgue measure zero. Introducing the projection $\Pi(x)$ of $x$ onto $\partial \Omega$ in the usual way, $\Sigma$ is also the set of points $x$ at which $\Pi(x)$ is not a singleton. So, if $\Pi(x)=\{\bar{x}\}$ for some $x \in \Omega$, then $d$ is differentiable at $x$ and

$$
D d(x)=\frac{x-\bar{x}}{|x-\bar{x}|} .
$$

REMARK 1 We recall that the distance function $d$ is the unique viscosity solution of the eikonal equation $|D u|=1$ in $\Omega$, with boundary condition $u=0$ in $\partial \Omega$. Equivalently, $d$ is the largest function such that $\|D u\|_{\infty, \Omega} \leq 1$ and $u=0$ on $\partial \Omega$.

For any $x \in \partial \Omega$ and $i=1, \ldots, n-1$, the number $\kappa_{i}(x)$ denotes the $i-t h$ principal curvature of $\partial \Omega$ at the point $x$, corresponding to a principal direction $e_{i}(x)$ orthogonal to $D d(x)$, with the sign convention $\kappa_{i} \geq 0$ if the normal section of $\Omega$ along the direction $e_{i}$ is convex. Also, we will label in the same way the extension of $\kappa_{i}$ to $\bar{\Omega} \backslash \Sigma$ given by

$$
\kappa_{i}(x)=\kappa_{i}(\Pi(x)) \quad \forall x \in \bar{\Omega} \backslash \Sigma .
$$

Denoting by $p \otimes q$ the tensor product of two vectors $p, q \in \mathbf{R}^{n}$, defined as

$$
(p \otimes q)(x)=p\langle q, x\rangle, \forall x \in \mathbf{R}^{n},
$$

for any $x \in \bar{\Omega}$ and any $y \in \Pi(x)$ we have

$$
\kappa_{i}(y) d(x) \leq 1 \quad \forall i=1, \ldots, n-1 .
$$

If, in addition, $x \in \bar{\Omega} \backslash \bar{\Sigma}$, then

$$
\kappa_{i}(x) d(x)<1 \quad \text { and } \quad D^{2} d(x)=-\sum_{i=1}^{n-1} \frac{\kappa_{i}(x)}{1-\kappa_{i}(x) d(x)} e_{i}(x) \otimes e_{i}(x)
$$

where $e_{i}(x)$ is the unit eigenvector corresponding to $\frac{\kappa_{i}(x)}{1-\kappa_{i}(x) d(x)}$.

We now turn our attention to the closure of $\Sigma$, a set that is also called the $c u t$ locus of $\partial \Omega$ in $\Omega$, and to the function

$$
\tau(x)=\left\{\begin{array}{cl}
\min \{t \geq 0: x+t D d(x) \in \bar{\Sigma}\} & \forall x \in \bar{\Omega} \backslash \bar{\Sigma} \\
0 & \forall x \in \bar{\Sigma}
\end{array}\right.
$$

Since the map $x \mapsto x+\tau(x) D d(x)$ is a natural retraction of $\bar{\Omega}$ onto $\bar{\Sigma}$, we will refer to $\tau(\cdot)$ as the maximal retraction length of $\Omega$ onto $\bar{\Sigma}$ or normal distance to $\bar{\Sigma}$. The regularity properties of $\tau$ are described by the following theorem due to Li and Nirenberg [19] (see also [18]).

THEOREM 2 Let $\Omega$ be a bounded domain in $\mathbf{R}^{n}$ with boundary of class $\mathcal{C}^{2,1}$. Then the map $\tau$ defined in (6) is Lipschitz continuous on $\partial \Omega$. 


\section{A representation formula for the solution}

Before stating a precise result for our problem, let us show a formal derivation of the representation formula for the solution in the case of $n=2$. Suppose $(u, v)$ is a smooth solution of (2). In view of Remark 1, we can take $u=d$. Moreover, suppose that $v$ vanishes on $\bar{\Sigma}$-this is reasonable from the point of view of the physical model, and can also be justified by a rigorous argument. Let us proceed to compute, for a given point $x \in \Omega \backslash \bar{\Sigma}$ and for any $t \in(0, \tau(x))$, the derivative

$$
\begin{aligned}
& \frac{d}{d t} v(x+t D d(x))=\langle D v(x+t D d(x), D d(x)\rangle \\
& \quad=-v(x+t D d(x)) \Delta d(x+t D d(x))-f(x+t D d(x))
\end{aligned}
$$

(recall that $D d(x+t D d(x))=D d(x))$. Now, observe that

$$
\Delta d(x+t D d(x))=-\frac{\kappa(x)}{1-(d(x)+t) \kappa(x)}
$$

since $\kappa(x+t D d(x))=\kappa(x)$ and $d(x+t D d(x))=d(x)+t$. Hence, $V(t):=$ $v(x+t D d(x))$ satisfies the Cauchy problem

$$
\left\{\begin{array}{l}
V^{\prime}(t)-\frac{\kappa(x)}{1-(d(x)+t) \kappa(x)} V(t)+f(x+t D d(x))=0 \\
V(\tau(x))=0 .
\end{array}\right.
$$

Thus, solving the above problem and noting that $v(x)=V(0)$, we conclude that, in $\Omega \backslash \bar{\Sigma}, v$ must be given by the formula

$$
v(x)=\int_{0}^{\tau(x)} f(x+t D d(x)) \frac{1-(d(x)+t) \kappa(x)}{1-d(x) \kappa(x)} d t \quad \forall x \in \Omega \backslash \bar{\Sigma}
$$

REMARK 3 We note that the above formula entails that $v$ vanishes at all points $x \in \Omega \backslash \bar{\Sigma}$ at which the half-line spanned by $D d(x)$ fails to intersect the support of $f$ before hitting $\bar{\Sigma}$. This description, which agrees with physical evidence, extends to dimension 2 the analogous result obtained in [17] for the one-dimensional case.

\section{Existence, uniqueness, regularity}

The following result, proved in [5] for $n=2$ and in [6] for $n \geq 2$, ensures the existence and uniqueness of the solution of (2), as well as a representation formula for such solution.

THEOREM 4 Let $\Omega \subset \mathbf{R}^{n}$ be a bounded domain with boundary of class $\mathcal{C}^{2}$ and $f \geq 0$ be a continuous function in $\Omega$. Then, a solution of system (2) is 
given by the pair $\left(d, v_{f}\right)$, where

$$
v_{f}(x)=\left\{\begin{array}{cl}
\int_{0}^{\tau(x)} f(x+t D d(x)) \prod_{i=1}^{n-1} \frac{1-(d(x)+t) \kappa_{i}(x)}{1-d(x) \kappa_{i}(x)} d t & \forall x \in \Omega \backslash \bar{\Sigma}, \\
0 & \forall x \in \bar{\Sigma},
\end{array}\right.
$$

where, $\kappa_{i}(x)$ denotes the $i-t h$ principal curvature of $\partial \Omega$ at the point $\Pi(x)$.

Moreover, the above solution is unique in the following sense: if $(u, v)$ is another solution of (2), then $v=v_{f}$ in $\Omega$, and $u=d$ in $\left\{x \in \Omega \mid v_{f}>0\right\}$.

A noteworthy aspect of the above result is that we do construct a continuous solution $v_{f}$, instead of just a measure or a function in $L^{1}(\Omega)$. So, Theorem 4 could also be viewed as a regularity result. Moreover, formula (7) can be used to derive further regularity properties. This will be the object of our next section.

\subsection{Regularity}

A natural question to ask is what kind of regularity one can expect for the solution $\left(d, v_{f}\right)$ of problem (2). For the first component, this is well understood: while $d$ is of class $\mathcal{C}^{2}$ on a neighborhood of $\partial \Omega$, the maximal regularity of $d$ in the whole domain $\bar{\Omega}$ is semiconcavity, a generalization of concavity preserving most of the local properties of concave functions (see [8] for a detailed description of such a class of functions).

On the other hand, the situation is different for the second component. Indeed, formula (7) suggests that the regularity of $v_{f}$ should depend on the regularity of $f$ and $\tau$. Therefore, our original problem leads to the question of the global regularity of the normal distance.

While it is easy to prove that $\tau$ is continuous on $\bar{\Omega}$, and locally Lipschitz in $\bar{\Omega} \backslash \bar{\Sigma}$ when $\partial \Omega \in \mathcal{C}^{2,1}$, the following example shows that $\tau$ may fail to be globally Lipschitz continous on $\bar{\Omega}$.

EXAMPLE 5 (THE PARABOLA CASE) In the cartesian plane consider the set $\Omega:=\left\{(x, y) \in \mathbf{R}^{2} \mid y>x^{2}\right\}$, whose boundary is a parabola with vertex $(0,0)$. By the symmetry of $\partial \Omega$ with respect to the vertical axis we deduce that $\bar{\Sigma}$ must be contained in such an axis. Moreover, $\bar{\Sigma}=\{(0, y) \mid y \geq 1 / 2\}$ and $\tau\left(\left(s, s^{2}\right)\right)=\frac{1}{2} \sqrt{1+4 s^{2}}$. Then, a straightforward computation shows that, for $a>0$ sufficiently small,

$$
|\tau((a, 1 / 2))-\tau((0,1 / 2))| \geq M|(a, 1 / 2)-(0,1 / 2)|^{2 / 3},
$$

for some $M>0$. So, $\tau$ cannot be Lipschitz continuous in the whole set $\Omega$.

On the positive side, we present two Hölder regularity results in $\mathbf{R}^{2}$ recently obtained in [7] - the former for $\tau$ the latter for $v_{f}$. 
THEOREM 6 Let $\Omega$ be abounded simply connected domain in $\mathbf{R}^{2}$ with analytic boundary, different from a disk. Then, there exists an integer $m \geq 2$ such that $\tau$ is Hölder continuous in $\Omega$ with exponent $\frac{2 m-2}{2 m-1}$.

In particular, $\tau$ is at least 2/3-Hölder continuous, and Example 5 describes the 'worst' possible case.

THEOREM 7 Assume that $f$ is a Lipschitz continuous function and that $\Omega$ is a simply connected bounded domain in $\mathbf{R}^{2}$ with analytic boundary, different from a disk. Then $v_{f}$ is a Hölder continuous function with exponent $\frac{1}{2 m-1}$ for some integer $m \geq 2$.

\section{Application to a variational problem}

We conclude this paper with an application to a problem in the calculus of variations which may seem quite unrelated to the present context at first glance. Let us consider an integral functional of the form

$$
J(u)=\int_{\Omega}[h(|D u|)-f(x) u] d x, \quad u \in W_{0}^{1,1}(\Omega)
$$

where $f \in L^{\infty}(\Omega)$ is a nonnegative function and $h:[0,+\infty) \rightarrow[0,+\infty]$ is a lower semicontinuous function (possibly with non-convex values) satisfying

$$
h(R)=0, \quad h(s) \geq \max \{0, \Lambda(s-R)\} \text { for some constants } R, \Lambda>0 .
$$

In a pioneering work, Cellina [11] proved that, if $\Omega$ is a convex domain (that is, an open bounded convex set) in $\mathrm{R}^{2}$ with piecewise smooth $\left(\mathcal{C}^{2}\right)$ boundary and $f \equiv 1$, then $J$ does attain its minimum in $W_{0}^{1,1}(\Omega)$, and a minimizer is explicitly given by the function

$$
u_{\Omega}(x)=R d(x), \quad x \in \Omega
$$

provided that the inradius $r_{\Omega}$ of $\Omega$ is small enough. (We recall that $r_{\Omega}$ is the supremum of the radii of all balls contained in $\Omega$.) This result has been extended to convex domains in $\mathbf{R}^{n}$ and to more general functionals in subsequent works (see $[9,10,13,21,22]$ ). One common point of all these results is that the set $\Omega$ is always a convex subset of $\mathbf{R}^{n}$. In this paper we will see that, using the representation formula (7), the function $u_{\Omega}$ defined in $(10)$ is a minimizer of $J$ in $W_{0}^{1,1}(\Omega)$, even on possibly nonconvex domains.

We say that a set $\Omega$ is a smooth $K$-admissible domain, $K \in \mathbf{R}$, if it is a connected open bounded subset of $\mathbf{R}^{n}$ with $\mathcal{C}^{2}$ boundary, such that the mean curvature of $\partial \Omega$ is bounded below by $K$, that is

$$
H(y):=\frac{1}{n-1} \sum_{i=1}^{n-1} \kappa_{i}(y) \geq K \quad \forall y \in \partial \Omega .
$$


We note that every connected bounded open set $\Omega \subset \mathrm{R}^{n}$ with $\mathcal{C}^{2}$ boundary is a $K$-admissible smooth domain for every $K$ satisfying

$$
K \leq \min _{y \in \partial \Omega} H(y)
$$

The following is a special case of a more general result obtained in [6].

THEOREM 8 Let $h:[0, \infty) \rightarrow[0, \infty]$ be a lower semicontinuous function satisfying (9), let $\Omega \subset \mathbf{R}^{n}$ be a smooth $K$-admissible domain, and let $f$ be a nonnegative Lipschitz continuous function in $\Omega$. If

$$
\|f\|_{\infty, \Omega} c\left(K, r_{\Omega}\right) \leq \Lambda
$$

where

$$
c\left(K, r_{\Omega}\right):=\frac{1-\left(1-K r_{\Omega}\right)^{n}}{n K} \text { if } K \neq 0, \quad c\left(K, r_{\Omega}\right):=r_{\Omega} \text { if } K=0,
$$

then the function $u_{\Omega}(x)=R d(x)$ is a minimizer in $W_{0}^{1,1}(\Omega)$ of the functional $J$ defined in (8).

We will now sketch the proof of Theorem 8 in order to point out the connection of this problem with (2). Given $f$ as above denote once again by $v_{f}$ the continuous function defined in (7). The first step of the proof, we will comment no further on in this context, consists of estimating the integrand in the representation formula for $v_{f}$ as in [6], to show that

$$
0 \leq v_{f}(x) \leq\|f\|_{\infty, \Omega} c\left(K, r_{\Omega}\right), \quad \forall x \in \Omega .
$$

Therefore, in view of assumption (11), we have

$$
0 \leq v_{f}(x) \leq \Lambda, \quad \forall x \in \Omega .
$$

Let $u \in W_{0}^{1,1}(\Omega)$. Since $h$ satisfies (9) and $v_{f}$ satisfies (14), we have that

$$
\begin{aligned}
h(|D u(x)|) & \geq v_{f}(x)(|D u(x)|-R) \\
& \geq h\left(\left|D u_{\Omega}(x)\right|\right)+v_{f}(x)\left\langle D d(x), D u(x)-D u_{\Omega}(x)\right\rangle,
\end{aligned}
$$

hence

$$
J(u) \geq J\left(u_{\Omega}\right)+\Delta,
$$

where

$$
\Delta=\int_{\Omega}\left[v_{f}(x)\left\langle D d(x), D u(x)-D u_{\Omega}(x)\right\rangle-f(x)\left(u(x)-u_{\Omega}(x)\right)\right] d x .
$$

Since $v_{f}$ is bounded, by a density argument equation (4) holds for every $\phi \in$ $W_{0}^{1,1}(\Omega)$. Choosing $\phi=u-u_{\Omega}$, we obtain that $\Delta$ vanishes, so that $J(u) \geq$ 
$J\left(u_{\Omega}\right)$. Since $u$ was an arbitrary function in $W_{0}^{1,1}(\Omega)$, we have proven that $u_{\Omega}$ is a minimizer of $J$ in $W_{0}^{1,1}(\Omega)$.

REMARK 9 We note:

1) The result of Theorem 8 can be extended to nonsmooth domains, such as domains satisfying a uniform exterior sphere condition. See [6] for details.

2) If $\Omega$ is a convex domain with smooth boundary, then condition

$$
L c\left(K, r_{\Omega}\right) \leq \Lambda
$$

is certainly satisfied provided that

$$
L r_{\Omega} \leq \Lambda
$$

Namely, it is enough to observe that a convex domain is a 0 -admissible domain, and that $c\left(0, r_{\Omega}\right)=r_{\Omega}$. Condition (15) was first introduced in [11] in the case of $f \equiv 1$. In [9] it was proven that, if (15) does not hold, then $J$ needs not have minimizers in $W_{0}^{1,1}(\Omega)$.

3) Assumption (11) of Theorem 8 for the existence of a minimizer of $J$ is optimal in the following sense. Let $h(s)=\max \{0, \Lambda(s-R)\}$ for some positive constants $\Lambda$ and $R$, let $f(x)=1$ and let $\Omega=B_{r}(0) \subset \mathbf{R}^{n}$. Then $r_{\Omega}=r$, and $\Omega$ is a $(1 / r)$-admissible domain. Since $c(1 / r, r)=r / n$, Theorem 8 states that the function $u_{\Omega}(x)=R d(x)$ is a minimizer of $J$ provided that $r \leq n \Lambda$. This condition is optimal: indeed, functional $J$ is not even bounded from below if $r>n \Lambda$. Let us define the sequence of functions in $W_{0}^{1,1}(\Omega)$

$$
u_{k}(x)= \begin{cases}k(r-|x|) & \text { if } n \Lambda<|x|<r \\ R(r-|x|) & \text { if }|x| \leq n \Lambda\end{cases}
$$

A straightforward computation shows that, for $k \geq R$,

$$
J\left(u_{k}\right)=\frac{\omega_{n}}{n+1}[\psi(n \Lambda)-\psi(r)] k+A,
$$

where $\omega_{n}$ is the $n$-dimensional Lebesgue measure of the unit ball of $\mathbf{R}^{n}, A$ is a constant independent of $k$, and $\psi(\rho)=\rho^{n+1}-(n+1) \Lambda \rho^{n}$. Since $\psi$ is strictly increasing for $\rho \geq n \Lambda$, and $r>n \Lambda$, we have that $\psi(n \Lambda)-\psi(r)<0$, hence $\lim _{k \rightarrow \infty} J\left(u_{k}\right)=-\infty$.

\section{References}

[1] L. Ambrosio. Optimal transport maps in Monge-Kantorovich problem. In Proceedings of the International Congress of Mathematicians Higher Ed. Press, 2002.

[2] G. Aronsson, L. C. Evans, Y. Wu. Fast/slow diffusion and growing sandpiles. J. Differential Equations 131:304-335, 1996. 
[3] T. Boutreux, P.-G. de Gennes. Surface flows of granular mixtures. I. General principles and minimal model. J. Phys. I France 6: 1295-1304, 1996.

[4] G. Bouchitté, G. Buttazzo, P. Seppechere. Shape optimization solutions via MongeKantorovich equation. C. R. Acad. Sci. Paris Ser. I Math. 324 10: 1185-1191, 1997.

[5] P. Cannarsa, P. Cardaliaguet. Representation of equilibrium solutions to the table problem for growing sandpile. J. Eur. Math. Soc. 6: 1-30, 2004.

[6] P. Cannarsa, P. Cardaliaguet, G. Crasta, E. Giorgieri. A boundary value problem for a PDE model in mass transfer theory: representation of solutions and applications. Calc. Var. DOI 10.1007/s00526-005-0328-7, 2005.

[7] P. Cannarsa, P. Cardaliaguet, E. Giorgieri. Hölder regularity of the normal distance with an application to a PDE model for growing sandpiles. Pre-print.

[8] P. Cannarsa, C. Sinestrari, Semiconcave functions, Hamilton-Jacobi equations, and optimal control. Birkhäuser progress in nonlinear differential equations and their applications. Boston, 2004.

[9] P. Celada, A. Cellina. Existence and non existence of solutions to a variational problem on a square. Houston J. Math. 24: 345-375, 1998.

[10] P. Celada, S. Perrotta, G. Treu. Existence of solutions for a class of non convex minimum problems. Math. Z. 228: 177-199, 1997.

[11] A. Cellina. Minimizing a functional depending on $\nabla u$ and on $u$. Ann. Inst. H. Poincaré, Anal. Non Linéaire 14:339-352, 1997.

[12] A. Cellina, S. Perrotta. On the validity of the maximum principle and of the Euler-Lagrange equation for a minimum problem depending on the gradient. SIAM J. Control Optim. 36: 1987-1998, 1997.

[13] G. Crasta, A. Malusa. Geometric constraints on the domain for a class of minimum problems. ESAIM Control Optim. Calc. Var. 9:125-133, 2003.

[14] L.C. Evans, M. Feldman, R. Gariepy. Fast/slow diffusion and growing sandpiles. J. Differential Equations 137:166-209, 1997.

[15] L.C. Evans, W. Gangbo. Differential equations methods for the Monge-Kantorovich mass transfer problem. Mem. Amer. Math. Soc. 137, no. 653, 1999.

[16] M. Feldman, R. J. McCann. Uniqueness and transport density in Monge's mass transportation problem. Calc. Var. 15:81-113, 2002.

[17] K. P. Hadeler, C. Kuttler. Dynamical models for granular matter. Granular Matter 2:9-18, 1999.

[18] J. Itoh, M. Tanaka. The Lipschitz continuity of the distance function to the cut locus. Trans. Am. Math. Soc. 353:21-40, 2001.

[19] Y. Y. Li, L. Nirenberg. The distance function to the boundary, Finsler geometry and the singular set of viscosity solutions of some Hamilton-Jacobi equations. Comm. Pure Appl. Math. 58:85-146, 2005.

[20] L. Prigozhin. Variational model of sandpile growth. European J. Appl. Math. 7:225-235, 1996.

[21] G. Treu. An existence result for a class of non convex problems of the Calculus of Variations. J. Convex Anal. 5:31-44, 1998.

[22] M. Vornicescu. A variational problem on subsets of $\mathrm{R}^{n}$. Proc. Roy. Soc. Edinburgh Sect. A 127:1089-1101, 1997. 